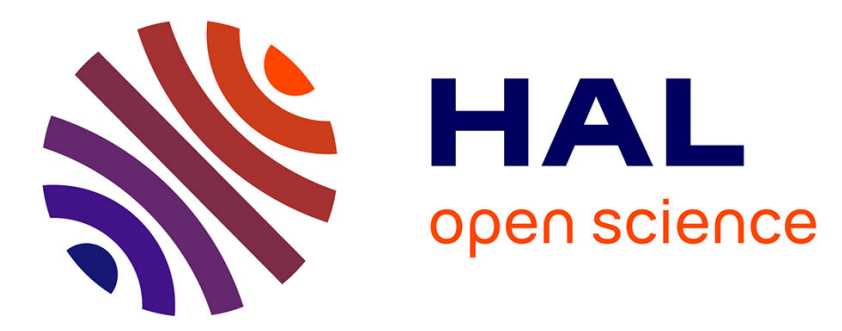

\title{
BGK source terms for out-of-equilibrium two-phase flow models \\ Olivier Hurisse
}

\section{- To cite this version:}

Olivier Hurisse. BGK source terms for out-of-equilibrium two-phase flow models. 2021. hal-03330954

\section{HAL Id: hal-03330954 \\ https://hal.science/hal-03330954}

Preprint submitted on 1 Sep 2021

HAL is a multi-disciplinary open access archive for the deposit and dissemination of scientific research documents, whether they are published or not. The documents may come from teaching and research institutions in France or abroad, or from public or private research centers.
L'archive ouverte pluridisciplinaire HAL, est destinée au dépôt et à la diffusion de documents scientifiques de niveau recherche, publiés ou non, émanant des établissements d'enseignement et de recherche français ou étrangers, des laboratoires publics ou privés. 


\title{
BGK source terms for out-of-equilibrium two-phase flow models
}

\author{
Olivier Hurisse, \\ EDF Lab Chatou, 6 quai Watier, 78400 Chatou, FRANCE.
}

September 2021

\begin{abstract}
A modified form of BGK source terms is proposed for modeling two-phase flows with thermodynamical disequilibrium. The novelty is that three independent time-scales allow to manage the return to the thermodynamical equilibrium while remaining in agreement with the second law of thermodynamics. This is achieved thanks to the definition of a "local-in-time" equilibrium state which tends towards the asymptotic equilibrium state when time increases. The thermodynamical paths of the system are then modified with respect to the classical BGK source terms used for two-phase flow modeling, and the relaxation process of the system towards the asymptotic equilibrium state can be defined with additional degrees of freedom. In a numerical point of view, both the classical and the modified BGK source terms have advantages. The choice between these two forms strongly depends on the numerical strategy used to perform simulations.
\end{abstract}

Keywords: Homogeneous model, Two-phase flows, Disequilibrium, BGK source terms.

MSC2020: 35Q31, 35Q79, 34A99, 34D20, 76T99. 


\section{Contents}

2 A homogeneous model accounting for the disequilibrium between the phases

3 The thermodynamical disequilibrium and the exchange between the phases 4

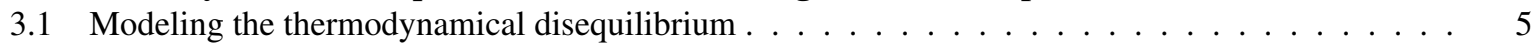

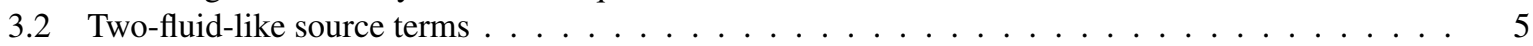

4 Classical BGK source terms 6

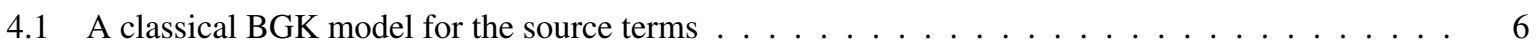

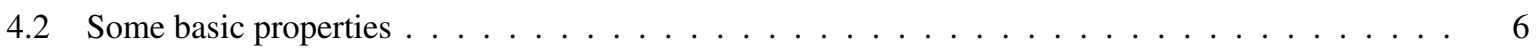

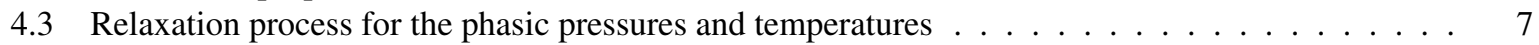

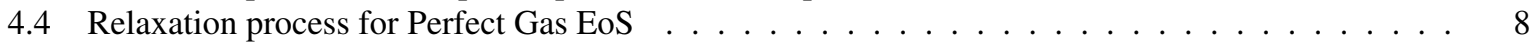

5 Proposition of modified BGK source terms $\quad 8$

5.1 BGK source terms involving three independent time-scales $\ldots \ldots \ldots \ldots \ldots$

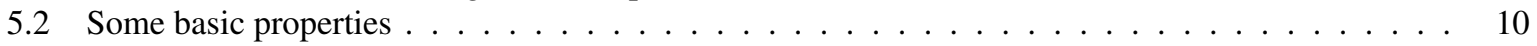

5.3 Relaxation process for the modified BGK source terms $\ldots \ldots \ldots \ldots \ldots \ldots$

6 Conclusion

\section{Introduction}

A wide range of two-phase flow models have been proposed in the literature in order to account for the thermodynamical disequilibrium between the phases, see for instance $[1,27,32,7,11,2]$ among many others. These models can be roughly split into two classes: the two-fluid models for which each phase possesses its own velocity field, and the homogeneous models assuming that both phases share the same velocity. In a thermodynamical point of view, the disequilibrium are taken into account in the systems of equations by the mean of source terms that rule the return to the thermodynamical equilibrium defined by the equality of the phasic pressures, the phasic temperatures and phasic chemical potentials. We focus herein on the source terms that take a BGK form (BGK stands for Bhatnagar-Gross-Krook). These form of source terms are classically used in Boltzmann methods for modeling collision terms. They are a linearized form of the exact terms for collisions that is highly non-linear. When dealing with two-phase flows, the BGK source terms are widely used in homogeneous models $[7,10,2,21,16]$. Besides, it should be noted that such source terms can also be used for two-fluid models [22, 24] and that other forms of source terms (i.e. not of BGK type) have been applied to homogeneous models as in [12, 13, 14].

The BGK source terms have two main advantages. Firstly, they are defined on the basis of an entropy for the mixture and they can thus easily be defined in agreement with the second law of thermodynamics. Secondly, the ODE system of equations associated with the BGK source terms is linear with respect to the primitive variables of the system. This allows to propose efficient and simple schemes on the basis of fractional step methods, as in $[21,16]$ for instance. The difficulty then lies in the computation of the thermodynamical equilibrium state that is defined by a system of three non-linear equations. An efficient scheme has been proposed for two-phase flows with general EoS (Equation Of State) $[8,16]$. With the latter, the $3 \times 3$ system is reduced to a single non-linear equation involving one unknown. Nevertheless, when turning to settings involving more than two phases this simplification is no more possible $[23,31]$ and computing the thermodynamical equilibrium becomes tricky.

In addition to this numerical difficulty for more than two phases, the classical BGK source terms are associated with a unique time scale for the return to the asymptotic equilibrium. This strongly couples the relaxation effect towards pressure, temperature and chemical potential equilibrium. Indeed, one could wish to manage separately these three relaxation processes by defining three different time-scales. The aim of the present work is to propose a modified form for the BGK source terms that enables to bypass the two drawback mentioned above. A new thermodynamical path is then proposed by using a "local-in-time" equilibrium state which tends towards the asymptotic thermodynamical equilibrium. This modified source terms agree with the second law of thermodynamics, whatever the three time-scales are. Moreover, the "local-in-time" thermodynamical equilibrium can be computed by solving three independent non-linear equations.

The outline of the paper is the following. In section 2, the homogeneous model proposed in [2] is shortly described and the link between the thermodynamical disequilibrium and the exchanges between the phases is recalled in section 3. Then the derivation of the classical BGK source terms is described in section 4 while modified BGK source terms are proposed in section 5. In these two sections, some properties of the source terms are given, even if only very basic ones can be proved. An effort has also been made in order to try to describe the pressure and temperature relaxation process for both sets of source terms. 


\section{A homogeneous model accounting for the disequilibrium between the phases}

The aim of the present section is not to present the complete derivation of the model proposed in [2]. This has been done in several references, see $[2,16,23,24,26,30,31]$ for instance. The model is introduced and its main properties are highlighted, in particular those associated with the exchanges between the phases. Some of these results will be useful in the following sections.

Let us consider two phases whose thermodynamical behaviors are described by their EoS, given through the phasic specific entropies $s_{k}, k=1,2$ :

$$
\left(\tau_{k}, e_{k}\right) \mapsto s_{k}\left(\tau_{k}, e_{k}\right),
$$

where $\tau_{k}$ and $e_{k}$ respectively stand for the specific volume and the specific energy. It is assumed that the entropies $s_{k}$ are in $C^{2}\left(\mathbb{R}_{*}^{+} \times \mathbb{R}_{*}^{+}\right)$, that they are strictly concave with respect to $\left(\tau_{k}, e_{k}\right)$, and that the partial derivative of $s_{k}$ with respect to the energy is strictly positive: $\partial_{e_{k}}\left(s_{k}\right)_{\tau_{k}}>0$. Moreover, it is assumed that within each of these phases the Gibbs relation holds:

$$
T_{k} d s_{k}=d e_{k}+P_{k} d \tau_{k}
$$

allowing to define the phasic pressure $P_{k}$ and the phasic temperature $T_{k}$ thanks to the entropy $s_{k}$ :

$$
\frac{1}{T_{k}}=\partial_{e_{k}}\left(s_{k}\right)_{\mid \tau_{k}}, \quad \text { and } \quad \frac{P_{k}}{T_{k}}=\partial_{\tau_{k}}\left(s_{k}\right)_{\mid e_{k}} .
$$

Following $[2,16,23,24,26,30,31]$, an immiscible mixture of these two phases is described by the mean of three fractions: the volume fraction $\alpha_{k}$ of phase $k$, the mass fraction $y_{k}$ of phase $k$ and the fraction $z_{k}$ of energy of phase $k$. Obviously, conservation of volume, mass and energy leads to the relations:

$$
\alpha_{1}+\alpha_{2}=1, \quad y_{1}+y_{2}=1 \quad \text { and } \quad z_{1}+z_{2}=1 .
$$

We assume here that the phasic EoS are such that these three fractions belong to $[0,1]$, see also remark 2 .

Remark $0 . \quad$ It should be noted that for miscible phases, when one assumes that both phases occupy the same volume, the volume fraction does not make sense and the volume conservation leads to $\alpha_{1}=\alpha_{2}=1$, see $[5,19$, $24,30,31]$.

By defining the mixture density $\rho$ and the mixture specific energy $e$ as:

$$
\rho=\alpha_{1} \rho_{1}+\alpha_{2} \rho_{2}, \quad \text { and } \quad \rho e=\alpha_{1} \rho_{1} e_{1}+\alpha_{2} \rho_{2} e_{2}
$$

with $\rho_{k}=1 / \tau_{k}$, the specific volume and the specific energy of phase $k$ can be obtained through the relations:

$$
\tau_{k}=\frac{\alpha_{k} \tau}{y_{k}}, \quad \text { and } \quad e_{k}=\frac{z_{k} \tau}{y_{k}}
$$

with $\tau=1 / \rho$. The specific mixture entropy $s$ for the mixture is defined as:

$$
\rho s=\alpha_{1} \rho_{1} s_{1}\left(\tau_{1}, e_{1}\right)+\alpha_{2} \rho_{2} s_{2}\left(\tau_{2}, e_{2}\right) .
$$

It can thus be written as a function of the fractions and the mixture specific volume and energy: $\left(\alpha_{1}, y_{1}, z_{1}, \tau, e\right) \mapsto$ $s\left(\alpha_{1}, y_{1}, z_{1}, \tau, e\right)$ by using relations (3) and (4). Moreover, thanks to the phasic Gibbs relations (1), a Gibbs relation for the mixture can be exhibited:

$$
T d s=d e+P d \tau+\tau\left(\frac{P_{1}}{T_{1}}-\frac{P_{2}}{T_{2}}\right) d \alpha_{1}-\left(\frac{\mu_{1}}{T_{1}}-\frac{\mu_{2}}{T_{2}}\right) d y_{1}+e\left(\frac{1}{T_{1}}-\frac{1}{T_{2}}\right) d z_{1},
$$

where $\mu_{k}=e_{k}+P_{k} \tau_{k}-T_{k} s_{k}$ is the chemical potential, and where the mixture pressure and the mixture temperature read:

$$
\frac{P}{T}=\alpha_{1} \frac{P_{1}}{T_{1}}+\alpha_{2} \frac{P_{2}}{T_{2}}, \quad \text { and } \quad \frac{1}{T}=z_{1} \frac{1}{T_{1}}+z_{2} \frac{1}{T_{2}}
$$

Remark 1. EoS may be defined in other thermodynamical planes, leading to different closures for the mixture pressure and temperature, see [9]. For instance, if one chooses the thermodynamical plane $\left(\tau_{k}, s_{k}\right)$, the Gibbs relation reads:

$$
d e_{k}\left(\tau_{k}, s_{k}\right)=T_{k} d s_{k}-P_{k} d \tau_{k}
$$

and thus we get:

$$
P_{k}=-\partial_{\tau_{k}}\left(e_{k}\right)_{\mid s_{k}} \quad \text { and } \quad T_{k}=\partial_{s_{k}}\left(e_{k}\right)_{\mid \tau_{k}}
$$


The Gibbs relation for the mixture then gives the following definitions for the mixture pressure and for the mixture temperature:

$$
P=\alpha_{1} P_{1}+\alpha_{2} P_{2} \text { and } T=\beta_{1} T_{1}+\beta_{2} T_{2},
$$

where $\beta_{k}=y_{k} s_{k} / s$ is the entropic fraction. More details may be found in [9].

The system of partial derivative equations used for simulating such mixtures is then based on the Euler system of equations. It is thus assumed that both phases have the same velocity field $U$. In one-dimensional setting, the system reads:

$$
\left\{\begin{array}{l}
\partial_{t}(\rho \mathscr{Y})+\partial_{x}(\rho U \mathscr{Y})=\rho \mathscr{S}, \\
\partial_{t}(\rho)+\partial_{x}(\rho U)=0, \\
\partial_{t}(\rho U)+\partial_{x}\left(\rho U^{2}+P\right)=0, \\
\partial_{t}(\rho E)+\partial_{x}(U(\rho E+P))=0,
\end{array}\right.
$$

where $\mathscr{Y}={ }^{t}\left(\alpha_{1}, y_{1}, z_{1}\right)$ is a vector containing the three fractions, $E=e+u^{2} / 2$ is the total energy of the mixture and $P$ is the pressure of the mixture as described in equation (6). The vector $\mathscr{S}={ }^{t}\left(\mathscr{S}_{\alpha_{1}}, \mathscr{S}_{y_{1}}, \mathscr{S}_{z_{1}}\right)$ gathers the source terms that rule the exchanges of volume, mass and energy between the two phases. It should be noted that in system (7) no external forces or heat exchange has been accounted for. We indeed intend to focus in this work on the source terms $\mathscr{S}$ which define the internal exchanges between the two phases.

Remark 2. It should be noted that system of equations (7) is strictly hyperbolic provided that the mixture temperature is strictly positive $[16,21,23]$. This is the case here if the phasic energies are positive. For the Generalized Stiffened Gas EoS, phasic energies may become negative, which might lead to negative energy fractions and to a negative mixture temperature $T$, even if the phasic temperature $T_{k}$ are positive. In such cases, hyperbolicity can be lost.

Thanks to (7), we have $d e+P d \tau=0$ so that equation (5) can be written:

$$
T d s=\tau\left(\frac{P_{1}}{T_{1}}-\frac{P_{2}}{T_{2}}\right) \mathscr{S}_{\alpha_{1}}-\left(\frac{\mu_{1}}{T_{1}}-\frac{\mu_{2}}{T_{2}}\right) \mathscr{S}_{y_{1}}+e\left(\frac{1}{T_{1}}-\frac{1}{T_{2}}\right) \mathscr{S}_{z_{1}},
$$

or equivalently, by reintroducing the mixture entropy:

$$
T d s=\partial_{\alpha_{1}}(s)_{\mid y_{1}, z_{1}, \tau, e} \mathscr{S}_{\alpha_{1}}+\partial_{y_{1}}(s)_{\mid \alpha_{1}, z_{1}, \tau, e} \mathscr{S}_{y_{1}}+\partial_{z_{1}}(s)_{\mid \alpha_{1}, y_{1}, \tau, e} \mathscr{S}_{z_{1}} .
$$

Equations (8) and (9) are equivalent since we have:

$$
\begin{gathered}
\partial_{\alpha_{1}}(s)_{\mid y_{1}, z_{1}, \tau, e}=\tau\left(\frac{P_{1}}{T_{1}}-\frac{P_{2}}{T_{2}}\right), \\
\partial_{y_{1}}(s)_{\mid \alpha_{1}, z_{1}, \tau, e}=-\left(\frac{\mu_{1}}{T_{1}}-\frac{\mu_{2}}{T_{2}}\right), \\
\partial_{z_{1}}(s)_{\mid \alpha_{1}, y_{1}, \tau, e}=e\left(\frac{1}{T_{1}}-\frac{1}{T_{2}}\right) .
\end{gathered}
$$

Both forms (8) and (9) will be useful in the following sections. Since $\mathscr{S}$ define exchanges between the two phases, they should agree with the second law of thermodynamics. When the two phasic entropies $\left(\tau_{k}, e_{k}\right) \mapsto s_{k}\left(\tau_{k}, e_{k}\right)$ are strictly concave, it has been proved in [26, 30, 21, 23] that $\left(\alpha_{1}, y_{1}, z_{1}\right) \mapsto s\left(\alpha_{1}, y_{1}, z_{1}, \tau, e\right)$ is strictly concave. Hence, in order to fulfill the second law of thermodynamics, the source terms $\mathscr{S}$ should be chosen so that the mixture entropy increases along a streamline:

$$
T d s \geq 0 .
$$

The following sections are dedicated to the study of possible source terms $\mathscr{S}$. We thus consider a closed mixture of two phases at rest and with a constant mixture energy $e$ and a constant mixture specific volume $\tau$. The whole model (7) has been presented for the sake of completeness. The following sections rely on equations (8)-(9) and the convective part of system (7) will not be considered.

\section{The thermodynamical disequilibrium and the exchange between the phases}

As mentioned at the end of the previous section, we consider here the source terms when the system composed of the mixture of the two phases is thermodynamically closed. That is: there is no mass exchange and no heat exchange between the mixture and its surrounding, and no external force acts on the mixture. This implies that $e$ and $\tau$ are constant. The time evolution of the entropy of the mixture is thus given by equation (8) or equation (9). Moreover, degenerate cases involving single phase situations are excluded from the present work: $\left(\alpha_{1}, y_{1}, z_{1}\right) \in$ ] $0,1\left[{ }^{3}\right.$, despite model (7) allows to handle single phase flows as shown in $[16,23]$. 
In the following, we then consider system of equations (7) at rest, i.e. $U=0$, and with constant $\tau$ and $e$. We get an ODE system whose variables are the fractions:

$$
\left\{\begin{array}{l}
d_{t} \mathscr{Y}=\mathscr{S} \\
d_{t} \rho=0, \\
U=0, \\
d_{t} e=0,
\end{array}\right.
$$

For the sake of readability, $\tau$ and $e$ will be omitted in the arguments of the different functions and the argument $\left(\alpha_{1}, y_{1}, z_{1}\right)$ will be replaced by $\mathscr{Y}$. Moreover, the source term vector $\mathscr{S}={ }^{t}\left(\mathscr{S}_{\alpha_{1}}, \mathscr{S}_{y_{1}}, \mathscr{S}_{z_{1}}\right)$ will be denoted by $\mathscr{S}={ }^{t}\left(\mathscr{S}_{1}, \mathscr{S}_{2}, \mathscr{S}_{2}\right)$.

\subsection{Modeling the thermodynamical disequilibrium}

Since the mixture entropy $\mathscr{Y} \mapsto s(\mathscr{Y})$ is strictly concave [26, 30, 21, 23], it possesses a unique maximum which is reached for a set of fractions:

$$
\overline{\mathscr{Y}}=\left(\bar{\alpha}_{1}, \bar{y}_{1}, \bar{z}_{1}\right),
$$

which only depends on $(\tau, e)$. The vector $\overline{\mathscr{Y}}$ is thus constant here and we get:

$$
\forall \mathscr{Y} \neq \overline{\mathscr{Y}}, s(\mathscr{Y})<\bar{s},
$$

where $\bar{s}$ denotes the maximum value of the mixture entropy: $\bar{s}=s(\overline{\mathscr{Y}})$. Since we have assumed that single phase flows do not occur, i.e. $\mathscr{Y} \in] 0,1[3$, then the first order conditions for the optimality of $\overline{\mathscr{Y}}$ hold:

$$
\left(\nabla_{\mathscr{Y} S}\right)(\overline{\mathscr{Y}})={ }^{t}(0,0,0) \text {. }
$$

Thanks to equations (10), (11) and (12), the latter can be expressed as pressure, temperature and chemical potential equilibrium:

$$
\begin{aligned}
& P_{1}(\overline{\mathscr{Y}})=P_{2}(\overline{\mathscr{Y}}), \\
& T_{1}(\overline{\mathscr{Y}})=T_{2}(\overline{\mathscr{Y}}), \\
& \mu_{1}(\overline{\mathscr{Y}})=\mu_{2}(\overline{\mathscr{Y}}) .
\end{aligned}
$$

Hence, for a given $(\tau, e)$, the fraction vector $\overline{\mathscr{Y}}$ is the unique vector that allows to recover the thermodynamical equilibrium defined by (14), (15) and (16). In other words, if the system is such that $\mathscr{Y} \neq \overline{\mathscr{Y}}$, then the associated thermodynamical state is out-of-equilibrium: at least one of the three equilibrium (14), (15) or (16) is not reached.

As mentioned in the previous section, the source terms $\mathscr{S}$ should be chosen in agreement with the second law of thermodynamics which states that $T d_{t} s \geq 0$. Since $\mathscr{Y} \mapsto s(\mathscr{Y})$ is strictly concave, this means that the time evolution of the thermodynamical system through (13) tends to increase the entropy $s$ and thus to decrease the gap between the state of the system $\mathscr{Y}$ and the thermodynamical equilibrium state $\overline{\mathscr{Y}}$. In that sense, the fractions $\mathscr{Y}$ characterize the thermodynamical disequilibrium between the phases and the source terms $\mathscr{S}$ determine the thermodynamical path of the system towards the equilibrium state defined by $\overline{\mathscr{Y}}$. Let us now study different ways for defining $\mathscr{S}$.

\subsection{Two-fluid-like source terms}

First of all, a short point is proposed in this section on a class of source terms that are classically used for two-fluid models, see among others $[1,15,25,6,11]$. They are not in a BGK form and they have been recently studied in the framework of homogeneous models in $[12,13,14]$. For these source terms, equation (8) should be considered. A natural way of defining $\mathscr{S}$ so that $T d_{t} s \geq 0$ is to choose:

$$
\begin{gathered}
\mathscr{S}_{\alpha_{1}}=\frac{K_{\alpha_{1}}}{\lambda_{\alpha_{1}}}\left(\frac{P_{1}}{T_{1}}-\frac{P_{2}}{T_{2}}\right), \\
\mathscr{S}_{y_{1}}=-\frac{K_{y_{1}}}{\lambda_{y_{1}}}\left(\frac{\mu_{1}}{T_{1}}-\frac{\mu_{2}}{T_{2}}\right), \\
\mathscr{S}_{z_{1}}=\frac{K_{z_{1}}}{\lambda_{z_{1}}}\left(\frac{1}{T_{1}}-\frac{1}{T_{2}}\right),
\end{gathered}
$$

where $K_{\alpha_{1}}, K_{y_{1}}$ and $K_{z_{1}}$ are normalization terms that should be positive and may depend on $\left(\alpha_{1}, y_{1}, z_{1}, \tau, e\right)$. The advantage of these source terms is that the three (positive) time scales $\lambda_{\alpha_{1}}, \lambda_{y_{1}}$ and $\lambda_{z_{1}}$ can be chosen independently and can depend on $\left(\alpha_{1}, y_{1}, z_{1}, \tau, e\right)$. This choice gives a lot of freedom in defining the paths followed by the system towards the equilibrium states. It will be seen in section (4) that the classical form of BGK source terms do not offer such a possibility.

Nevertheless, source terms of the form (17)-(19) have a known drawback when turning to numerical simulation. Building efficient and robust schemes for these source terms can be tricky, see for instance [12, 18, 17, 29, 28, 3, $4,24]$. When considering two-fluid models, BGK-like source terms have been recently proposed in order to avoid such difficulties $[22,24]$. Indeed, it will be seen in the next section that the form of the BGK source terms render their numerical discretization easier. 


\section{Classical BGK source terms}

In this section, classical BGK source terms associated with model (7) are first studied. They have been proposed in [2] and they strongly rely on the property of concavity of the mixture entropy.

\subsection{A classical BGK model for the source terms}

The classical form of source terms for model (7) relies on the Gibbs relation (9) and on the remark that the mixture entropy $\mathscr{Y} \mapsto s(\mathscr{Y})$ is strictly concave. Indeed, the concavity of $s$ implies that the plane tangent to $s$ at a point $\mathscr{Y}$ is always above $s$. In other words, for all $\mathscr{Y}=\left(\alpha_{1}, y_{1}, z_{1}\right)$, we have:

$$
\forall \mathscr{Y}^{\prime}={ }^{t}\left(\alpha_{1}^{\prime}, y_{1}^{\prime}, z_{1}^{\prime}\right), \quad s\left(\mathscr{Y}^{\prime}\right) \leq s(\mathscr{Y})+{ }^{t}\left(\nabla_{\mathscr{Y}} s\right)(\mathscr{Y}) \cdot\left(\mathscr{Y}^{\prime}-\mathscr{Y}\right) .
$$

So that, setting $\mathscr{Y}^{\prime}=\overline{\mathscr{Y}}$ we get thanks to the definition of $\overline{\mathscr{Y}}$ that for all $\mathscr{Y}$ :

$$
0 \leq \bar{s}-s(\mathscr{Y})=s(\overline{\mathscr{Y}})-s(\mathscr{Y}) \leq{ }^{t}\left(\nabla_{\mathscr{Y}} s\right)(\mathscr{Y}) \cdot(\overline{\mathscr{Y}}-\mathscr{Y}) .
$$

Equation (9) can be written:

$$
T d_{t} s={ }^{t}\left(\nabla_{\mathscr{Y}} s\right)(\mathscr{Y}) \cdot \mathscr{S},
$$

hence, by choosing $\mathscr{S}=(\overline{\mathscr{Y}}-\mathscr{Y}) / \lambda$, with $\lambda$ a positive time-scale, we obtain from the previous inequality that:

$$
T d_{t} s={ }^{t}\left(\nabla_{\mathscr{Y}} s\right)(\mathscr{Y}) \cdot(\overline{\mathscr{Y}}-\mathscr{Y}) / \lambda \geq 0
$$

We can conclude that the source terms defined as:

$$
\mathscr{S}=\frac{\overline{\mathscr{Y}}-\mathscr{Y}}{\lambda}, \quad \overline{\mathscr{Y}}=\underset{\mathscr{Y}}{\operatorname{argmax}}(s(\mathscr{Y})),
$$

comply with the second law of thermodynamics.

It is an important point to be quoted that, on the contrary to the source terms of section 3.2, source terms (21) only involve a unique time scale $\lambda$ for all the fractions.

\subsection{Some basic properties}

System (13) associated with BGK source terms (21) is a quasi-linear set of ODE which and can be easily integrated. Indeed, since $\overline{\mathscr{Y}}$ only depends on $(\tau, e)$, it is constant. Thus we get for each component $\mathscr{Y}_{i}, i=1 . .3$, of the vector Y:

$$
\mathscr{Y}_{i}(t)=\overline{\mathscr{Y}}_{i}+\left(\mathscr{Y}_{i}(0)-\overline{\mathscr{Y}}_{i}\right) \exp \left(-\int_{0}^{t} \frac{d \zeta}{\lambda(\zeta)}\right) .
$$

Several very basic properties arise from formula (22).

First, since $\lambda>0$, equation (22) states that:

$$
\forall t \geq 0, \quad \min \left(\mathscr{Y}_{i}(0), \overline{\mathscr{Y}}_{i}\right) \leq \mathscr{Y}_{i}(t) \leq \max \left(\mathscr{Y}_{i}(0), \overline{\mathscr{Y}}_{i}\right)
$$

If we assume that the initial fraction $\mathscr{Y}_{i}(0)$ and the equilibrium fraction $\overline{\mathscr{Y}}_{i}$ belong to $] 0,1[$, the inequalities above mean that $t \mapsto \mathscr{Y}_{i}(t)$ is bounded and remains in $] 0,1\left[\right.$ for all $t \geq 0$. Moreover, (22) implies that $\mathscr{Y}_{i}(t)$ is an increasing (resp. decreasing) function of $t$ if $\mathscr{Y}_{i}(0) \geq \overline{\mathscr{Y}}_{i}$ (resp. $\left.\mathscr{Y}_{i}(0) \leq \overline{\mathscr{Y}}_{i}\right)$.

Let us now focus on the integral term in relation (22). If its limit tends to $+\infty$ :

$$
\int_{0}^{t} \frac{d \zeta}{\lambda(\zeta)} \underset{t \rightarrow+\infty}{\longrightarrow}+\infty
$$

then the exponential term in (22) tends to zero when $t \rightarrow+\infty$. In such cases, the equilibrium state is asymptotically reached by the system:

$$
\lim _{t \rightarrow+\infty} \mathscr{Y}_{i}(t)=\overline{\mathscr{Y}}_{i}, \quad i=1 . .3 .
$$

This is for instance the case when for all $t \geq 0$ the time-scale remains bounded. On the contrary, if the integral remains bounded the system tends to a limit state that is different from the equilibrium state. The latter is thus not asymptomatically reached. Such situations occur for instance when the relaxation time-scale is a function that "quickly" increases to $+\infty$. If one chooses for instance: $\lambda(t)=\exp (t)$, the system tends to:

$$
\lim _{t \rightarrow+\infty} \mathscr{Y}_{i}(t)=\overline{\mathscr{Y}}_{i}+\left(\mathscr{Y}_{i}(0)-\overline{\mathscr{Y}}_{i}\right) \exp (-1) \neq \overline{\mathscr{Y}}_{i}, \quad i=1 . .3 .
$$

For such time-scales, the relaxation of the fraction to the equilibrium fraction is partial and the thermodynamical equilibrium can not be reached.

These remarks clearly show that the equilibrium state defined by $\overline{\mathscr{Y}}$ is a stable equilibrium state for system (13). It is asymptotically reached when condition (23) is fulfilled by the relaxation time-scale.

Remark 3. In fact, a Lyapunov function can be built for system (13) on the basis of the mixture entropy, $\mathscr{L}: \mathscr{Y} \mapsto$ $\bar{s}-s(\mathscr{Y})$. Such a Lyapunov function has been used in [14] in order to study the stability of the source terms of section 3.2. Indeed, system (13) associated with these source terms leads to a highly non-linear system which can not be studied as easily as system (13) associated with BGK source terms (21). 


\subsection{Relaxation process for the phasic pressures and temperatures}

It has been shown in section (4.2) that the fractions $\mathscr{Y}$ exponentially relax to the equilibrium fractions $\overline{\mathscr{Y}}$ provided that condition (23) holds for the time scale. The equilibrium state is clearly defined as the unique state for which pressure, temperature and chemical potential of the two phases are equal, see section 3.1. So it seems natural to study how the relaxation process for the fractions translates in terms of phasic pressures and temperatures. For the sake of simplicity, relaxation for chemical potentials will not be studied here. The latter is associated with the mass fraction through relation (11). We then consider the specific case where mass fractions $y_{k}$ are constant. Typically, if one sets $y_{1}(0)=\bar{y}_{1}$, equation (22) states that for all $t \geq, y_{1}(t)=y_{1}(0)=\bar{y}_{1}$ and that $d_{t} y_{1}=0$.

Let us consider a function $\phi_{k}$ defined for phase $k: \phi_{k}:\left(\tau_{k}, e_{k}\right) \mapsto \phi_{k}\left(\tau_{k}, e_{k}\right)$. Thanks to system (13), we get that its derivative is:

$$
d_{t} \phi_{k}=\partial_{\tau_{k}}\left(\phi_{f}\right) \frac{\tau_{k}}{\alpha_{k}} d_{t} \alpha_{k}-\left(\partial_{\tau_{k}}\left(\phi_{f}\right) \frac{\tau_{k}}{y_{k}}+\partial_{e_{k}}\left(\phi_{f}\right) \frac{e_{k}}{y_{k}}\right) d_{t} y_{k}+\partial_{e_{k}}\left(\phi_{f}\right) \frac{e_{k}}{z_{k}} d_{t} z_{k},
$$

which for constant mass fraction can be simplified in:

$$
d_{t} \phi_{k}=\partial_{\tau_{k}}\left(\phi_{f}\right) \frac{\tau_{k}}{\alpha_{k}} d_{t} \alpha_{k}+\partial_{e_{k}}\left(\phi_{f}\right) \frac{e_{k}}{z_{k}} d_{t} z_{k}
$$

By setting successively $\phi_{k}=1 / T_{k}=\partial_{e_{k}}\left(s_{k}\right)_{\mid \tau_{k}}$ and $\phi_{k}=P_{k} / T_{k}=\partial_{\tau_{k}}\left(s_{k}\right)_{\mid e_{k}}$ in equation (24), we get that:

$$
\left(\begin{array}{c}
d_{t}\left(P / T_{k}\right) \\
d_{t}\left(1 / T_{k}\right)
\end{array}\right)=\frac{1}{y_{k}} \nabla_{\left(\tau_{k}, e_{k}\right)}^{2} s_{k}\left(\begin{array}{c}
\tau d_{t} \alpha_{k} \\
e d_{t} z_{k}
\end{array}\right)
$$

where $\nabla_{\left(\tau_{k}, e_{k}\right)}^{2} s_{k}$ stands for the Hessian matrix of the phasic entropy $\left(\tau_{k}, e_{k}\right) \mapsto s_{k}\left(\tau_{k}, e_{k}\right)$. By combining equation (25) for the two phases, we easily get the relation:

$$
\left(\begin{array}{c}
d_{t}\left(P_{1} / T_{1}-P_{2} / T_{2}\right) \\
d_{t}\left(1 / T_{1}-1 / T_{2}\right)
\end{array}\right)=\left(\frac{1}{y_{1}} \nabla_{\left(\tau_{1}, e_{1}\right)}^{2} s_{1}+\frac{1}{y_{2}} \nabla_{\left(\tau_{2}, e_{2}\right)}^{2} s_{2}\right)\left(\begin{array}{c}
\tau d_{t} \alpha_{1} \\
e d_{t} z_{1}
\end{array}\right),
$$

or using the definition of the source terms (21):

$$
\left(\begin{array}{c}
d_{t}\left(P_{1} / T_{1}-P_{2} / T_{2}\right) \\
d_{t}\left(1 / T_{1}-1 / T_{2}\right)
\end{array}\right)=\left(\frac{1}{y_{1}} \nabla_{\left(\tau_{1}, e_{1}\right)}^{2} s_{1}+\frac{1}{y_{2}} \nabla_{\left(\tau_{2}, e_{2}\right)}^{2} s_{2}\right)\left(\begin{array}{c}
\frac{\tau\left(\bar{\alpha}_{1}-\alpha_{1}\right)}{\lambda} \\
\frac{e\left(\bar{z}_{1}-z_{1}\right)}{\lambda}
\end{array}\right) .
$$

Since the phasic entropies are strictly concave with respect to $\left(\tau_{k}, e_{k}\right)$ and since the mass fractions are constant, it implies that the matrix involving the Hessian matrices on the right hand side of (26) and (27) is symmetric definite strictly negative, we thus get the inequality:

$$
t\left(\begin{array}{c}
d_{t}\left(P_{1} / T_{1}-P_{2} / T_{2}\right) \\
d_{t}\left(1 / T_{1}-1 / T_{2}\right)
\end{array}\right) \cdot\left(\begin{array}{c}
\frac{\tau\left(\bar{\alpha}_{1}-\alpha_{1}\right)}{\lambda} \\
\frac{e\left(\bar{z}_{1}-z_{1}\right)}{\lambda}
\end{array}\right)<0
$$

Moreover, for source terms (21), equation (20) gives:

$$
{ }^{t}\left(\nabla_{\mathscr{Y} S}\right)(\mathscr{Y}) \cdot(\overline{\mathscr{Y}}-\mathscr{Y}) \geq 0
$$

By using formulae (10)-(12) for the derivatives of the mixture entropy $s$ and by setting $y_{1}=\bar{y}_{1}$ in (29), we obtain the inequality:

$$
t\left(\begin{array}{c}
\left(P_{1} / T_{1}-P_{2} / T_{2}\right) \\
\left(1 / T_{1}-1 / T_{2}\right)
\end{array}\right) \cdot\left(\begin{array}{c}
\frac{\tau\left(\bar{\alpha}_{1}-\alpha_{1}\right)}{\lambda} \\
\frac{e\left(\bar{z}_{1}-z_{1}\right)}{\lambda}
\end{array}\right) \geq 0
$$

From equation (22), we know that $W$, the second vector on the left hand side of inequalities (30) and (28), can be written as a constant vector $W_{0}$ multiplied by a function of time $\omega(t), W=\omega(t) W_{0}$, where we have:

$$
W=\left(\begin{array}{c}
\frac{\tau\left(\bar{\alpha}_{1}-\alpha_{1}\right)}{\lambda} \\
\frac{e\left(\bar{z}_{1}-z_{1}\right)}{\lambda}
\end{array}\right), \quad W_{0}=\left(\begin{array}{c}
\tau\left(\bar{\alpha}_{1}-\alpha_{1}(0)\right) \\
e\left(\bar{z}_{1}-z_{1}(0)\right)
\end{array}\right), \text { and } \quad \omega=\frac{1}{\lambda} \exp \left(-\int_{0}^{t} \frac{d \zeta}{\lambda(\zeta)}\right) .
$$

If at time $t=0$ the system is out of equilibrium, then $W_{0} \neq{ }^{t}(0,0)$ and $W_{0}$ allows to define two complementary half spaces of $\mathbb{R}^{2}$ :

$$
\mathscr{W}^{+}=\left\{(x, y) \in \mathbb{R}^{2},{ }^{t}(x, y) \cdot W_{0} \geq 0\right\} \text { and } \mathscr{W}^{-}=\left\{(x, y) \in \mathbb{R}^{2},{ }^{t}(x, y) \cdot W_{0}<0\right\} .
$$

The vector of pressure and temperature differences, ${ }^{t}\left(P_{1} / T_{1}-P_{2} / T_{2}, 1 / T_{1}-1 / T_{2}\right)$, lies in $\mathscr{W}^{+}$, and its derivative with time lies in $\mathscr{W}^{-}$.

At that point it seems difficult to go further in the analysis of the relaxation process. Indeed, it depends on the phasic EoS and on the link between $W_{0}$ and the initial pressure and temperature differences. In the next section, the example of perfect gas EoS is developed. For these simple EoS, $W_{0}$ is co-linear with the initial pressure and temperature differences, and the analysis of the relaxation process can be performed further quite easily. 


\subsection{Relaxation process for Perfect Gas EoS}

In the very particular case of two Perfect Gas EoS, it can be proven that the pressure and temperature relaxation is monotonic when considering that mass transfer does not occur. For a Perfect Gas EoS, we have the entropy:

$$
s_{k}\left(\tau_{k}, e_{k}\right)=C_{v, k} \ln \left(e_{k} \tau_{k}^{\left(\gamma_{k}-1\right)}\right)+s_{0, k}
$$

where $C_{v, k}>0, \gamma_{k}>1$ and $s_{0, k}$ are parameters. The pressure and temperature (2) are then: $P_{k}=\left(\gamma_{k}-1\right) e_{k} / \tau_{k}$ and $T_{k}=e_{k} / C_{v, k}$, and thus $P_{k} / T_{k}=\left(\gamma_{k}-1\right) C_{v, k} / \tau_{k}$. Hence, $1 / T_{k}$ only depends on $e_{k}$ and $P_{k} / T_{k}$ only depends on $\tau_{k}$, which means that the matrix on the right hand side of system of equations (27) is diagonal. In fact we have:

$$
\left\{\begin{aligned}
d_{t}\left(P_{1} / T_{1}-P_{2} / T_{2}\right) & =-\left(\frac{1}{z_{1} T_{1}}+\frac{1}{z_{2} T_{2}}\right) \frac{\left(\bar{\alpha}_{1}-\alpha_{1}\right)}{\lambda} \\
d_{t}\left(1 / T_{1}-1 / T_{2}\right) & =-\left(\frac{P_{1}}{\alpha_{1}}+\frac{P_{2}}{\alpha_{2}}\right) \frac{\left(\bar{z}_{1}-z_{1}\right)}{\lambda}
\end{aligned}\right.
$$

Moreover, the volume and energy equilibrium fractions can be written explicitly from the temperature and pressure equilibrium (14)-(15):

$$
\bar{\alpha}_{1}=\frac{\bar{y}_{1} \delta_{1}}{\bar{y}_{1} \delta_{1}+\bar{y}_{2} \delta_{2}} \quad \text { and } \quad \bar{z}_{1}=\frac{\bar{y}_{1} C_{v, 1}}{\bar{y}_{1} C_{v, 1}+\bar{y}_{2} C_{v, 2}}
$$

where $\delta_{k}=\left(\gamma_{k}-1\right) C_{v, k}$. We recall that we consider here the case $y_{k}=\bar{y}_{k}$, so that in fact $\bar{\alpha}_{1}$ and $\bar{z}_{1}$ - and thus $\overline{\mathscr{Y}}$ do not depend on $\mathscr{Y}$. After some easy computations using these explicit formulae, it can be obtained that:

$$
\bar{\alpha}_{1}-\alpha_{1}=\frac{T}{P} \alpha_{1} \alpha_{2}\left(\frac{P_{1}}{T_{1}}-\frac{P_{2}}{T_{2}}\right)
$$

and

$$
\bar{z}_{1}-z=T z_{1} z_{2}\left(\frac{1}{T_{1}}-\frac{1}{T_{2}}\right)
$$

which combined with system (31) ensures that the time evolution of $\left(P_{1} / T_{1}-P_{2} / T_{2}\right)$ and $\left(1 / T_{1}-1 / T_{2}\right)$ is monotonic.

Remark 4. The equilibrium mass fraction $\bar{y}_{1}$ is defined thanks to the chemical potential equilibrium (16). The latter is non-linear with respect to $\tau_{k}$ and $e_{k}$ even for Perfect Gas EoS since: $\mu_{k} / T_{k}=\gamma_{k} C_{V, k}-s_{k}\left(\tau_{k}, e_{k}\right)$. Thus $\bar{y}_{1}$ is such that:

$$
\gamma_{1} C_{V, 1}-s_{1}\left(\frac{\bar{\alpha}_{1} \tau}{\bar{y}_{1}}, \frac{\bar{z}_{1} e}{\bar{y}_{1}}\right)=\gamma_{2} C_{V, 2}-s_{2}\left(\frac{\left(1-\bar{\alpha}_{1}\right) \tau}{\left(1-\bar{y}_{1}\right)}, \frac{\left(1-\bar{z}_{1}\right) e}{\left(1-\bar{y}_{1}\right)}\right) .
$$

The source term for the mass fraction $\mathscr{S}_{2}$ can then not be written in terms of the difference of the potential, as it is the case for $\mathscr{S}_{1}$ and $\mathscr{S}_{3}$ which can respectively be written in terms of the differences $P_{1} / T_{1}-P_{2} / T_{2}$ and $1 / T_{1}-1 / T_{2}$.

\section{Proposition of modified BGK source terms}

The source terms of section 3.2 allow to define independent time-scales for pressure, temperature and chemical potential relaxation. In a physical point of view, it is an interesting feature. Indeed, this enables more possibilities in the definition of the thermodynamical paths towards the equilibrium state. In this section, the BGK source terms of section 4 are modified in order to account for three independent time-scales for volume, mass and energy fractions.

\subsection{BGK source terms involving three independent time-scales}

A first natural way of introducing three time-scales in BGK source terms could be to modify source terms (21) into:

$$
\mathscr{S}_{i}=\frac{\overline{\mathscr{Y}}_{i}-\mathscr{Y}_{i}}{\lambda_{i}}, \quad \overline{\mathscr{Y}}=\underset{\mathscr{Y}}{\operatorname{argmax}}(s(\mathscr{Y})),
$$

with $\lambda_{i}>0$ for $i=1 . .3$. In section 4 , choosing a single time-scale, $\lambda_{i}=\lambda$, ensures that the source terms comply with the second law of thermodynamics whatever the form for $\lambda>0$ is. With choice (34), this is not the case because the concavity of the mixture entropy can not be used as in section 4.1 . Hence, for each choices of $\lambda_{i}$ one should check if the second law of thermodynamics is fulfilled. This might be very tricky. An other suggestion is thus made here, and it also relies on the concavity of the mixture entropy as in section 4 . The basic idea is very close to the one proposed in [22]. 
Since the mixture entropy $\mathscr{Y} \mapsto s(\mathscr{Y})$ is strictly concave with respect to $\mathscr{Y}$, the three functions:

$$
\begin{gathered}
\eta_{1}: \alpha_{1} \mapsto s\left(\alpha_{1}, y_{1}, z_{1}\right), \\
\eta_{2}: y_{1} \mapsto s\left(\alpha_{1}, y_{1}, z_{1}\right), \\
\eta_{3}: z_{1} \mapsto s\left(\alpha_{1}, y_{1}, z_{1}\right),
\end{gathered}
$$

are also strictly concave. For the function $\eta_{i}$, the variable is $\mathscr{Y}_{i}$ and $\mathscr{Y}_{j}, j \neq i$, are parameters. In the following, an abuse of notation is used: parameters $\mathscr{Y}_{j}, j \neq i$ are omitted in the arguments of $\eta_{i}$. Let us define the maximum of each of these functions by:

$$
\widetilde{\eta}_{i}=\eta_{i}\left(\widetilde{\mathscr{Y}_{i}}\right)=\max _{\mathscr{Y}_{i}}\left(\eta_{i}\left(\mathscr{Y}_{i}\right)\right), i=1 . .3
$$

In the definition (38) of the maximum for $\eta_{i}$, the quantities $\mathscr{Y}_{j}, j \neq i$, are fixed. It is an important point to be quoted that in general we have:

$$
\overline{\mathscr{Y}} \neq \widetilde{\mathscr{Y}}={ }^{t}\left(\widetilde{\mathscr{Y}_{1}}, \widetilde{\mathscr{Y}} 2, \widetilde{\mathscr{Y}} 3\right)
$$

Indeed, the equilibrium fraction vector $\overline{\mathscr{Y}}$ of section 4 is a constant, it does not depend of the fractions $\mathscr{Y}_{i}$. On the contrary, the equilibrium fraction vector defined by (38) depends on the fractions and it is thus not constant - but it does not depend on $\tau$ and $e$-: $\mathscr{Y} \mapsto \widetilde{\mathscr{Y}}(\mathscr{Y})$. More precisely, the component $i$ of $\widetilde{\mathscr{Y}}$ depends on the two components $j \neq i$ of vector $\mathscr{Y}$.

Since the functions $\eta_{i}$ are strictly concave with respect to $\mathscr{Y}_{i}$, the recipes used in section 4.1 can be applied here. For all $\mathscr{Y}_{i}$, we have:

$$
\forall \mathscr{Y}_{i}^{\prime}, \eta_{i}\left(\mathscr{Y}_{i}^{\prime}\right) \leq \eta_{i}\left(\mathscr{Y}_{i}\right)+\frac{\partial \eta_{i}}{\partial \mathscr{Y}_{i}}\left(\mathscr{Y}_{i}\right) \times\left(\mathscr{Y}_{i}^{\prime}-\mathscr{Y}_{i}\right)
$$

and if we choose $\mathscr{Y}_{i}^{\prime}=\widetilde{\mathscr{Y}}_{i}$ we obtain for all $\mathscr{Y}_{i}$ :

$$
0 \leq \widetilde{\eta}_{i}-\eta_{i}\left(\mathscr{Y}_{i}\right) \leq \frac{\partial \eta_{i}}{\partial \mathscr{Y}_{i}}\left(\mathscr{Y}_{i}\right) \times\left(\widetilde{\mathscr{Y}}_{i}-\mathscr{Y}_{i}\right)
$$

Moreover, thanks to equations (10)-(12) we easily get the derivatives of $\eta_{i}$ with respect to $\mathscr{Y}_{i}$ :

$$
\begin{gathered}
\frac{\partial \eta_{1}}{\partial \alpha_{1}}=\partial_{\alpha_{1}}(s)_{\mid y_{1}, z_{1}}=\tau\left(\frac{P_{1}}{T_{1}}-\frac{P_{2}}{T_{2}}\right)(\mathscr{Y}), \\
\frac{\partial \eta_{2}}{\partial y_{1}}=\partial_{y_{1}}(s)_{\mid \alpha_{1}, z_{1}}=-\left(\frac{\mu_{1}}{T_{1}}-\frac{\mu_{2}}{T_{2}}\right)(\mathscr{Y}), \\
\frac{\partial \eta_{3}}{\partial z_{1}}=\partial_{z_{1}}(s)_{\mid \alpha_{1}, y_{1}}=e\left(\frac{1}{T_{1}}-\frac{1}{T_{2}}\right)(\mathscr{Y}) .
\end{gathered}
$$

As a consequence, source terms in agreement with the second law of thermodynamics can be defined on the basis of $\widetilde{\mathscr{Y}}$ as:

$$
\mathscr{S}_{i}=\frac{\widetilde{\mathscr{Y}_{i}}(\mathscr{Y})-\mathscr{Y}_{i}}{\lambda_{i}}, \quad \widetilde{\mathscr{Y}_{i}}(\mathscr{Y})=\underset{\mathscr{Y}_{i}}{\operatorname{argmax}}\left(\eta_{i}\left(\mathscr{Y}_{i}\right)\right),
$$

with independent time scales $\lambda_{i}>0$. Indeed, with (42) each term on the right hand side of Gibbs relation (9) is positive, whatever the time scale $\lambda_{i}>0$ is. As the system of section 4.1, system of ODE (13) associated with source terms (42) is a non-linear system.

At last, when the thermodynamical equilibrium is reached for $0<\widetilde{\mathscr{Y}}_{i}<1$, the fractions are defined by the optimality constraint for each function $\eta_{i}$ that leads to:

$$
\begin{aligned}
\frac{P_{1}}{T_{1}}\left(\widetilde{\alpha}_{1}, y_{1}, z_{1}\right) & =\frac{P_{2}}{T_{2}}\left(\widetilde{\alpha}_{1}, y_{1}, z_{1}\right), \\
\frac{\mu_{1}}{T_{1}}\left(\alpha_{1}, \widetilde{y}_{1}, z_{1}\right) & =\frac{\mu_{2}}{T_{2}}\left(\alpha_{1}, \widetilde{y}_{1}, z_{1}\right), \\
\frac{1}{T_{1}}\left(\alpha_{1}, y_{1}, \widetilde{z}_{1}\right) & =\frac{1}{T_{2}}\left(\alpha_{1}, y_{1}, \widetilde{z}_{1}\right) .
\end{aligned}
$$

The equilibrium fractions $\widetilde{\mathscr{Y}_{i}}$ may not remain in $[0,1]$. The reason is the same than for the classical BGK model as described in remark 2, and it depends on the choice of the phasic EoS. Nevertheless, in the following it will be assumed that $\widetilde{\mathscr{Y}}$ belongs to $[0,1]^{3}$. 


\subsection{Some basic properties}

As mentioned previously, ODE system (13) with source terms (42) is non-linear and its study is more complex than the system of section 4. First of all, it has been assumed in section 2 that the phasic entropies belong to $C^{2}\left(\mathbb{R}_{*}^{+} \times \mathbb{R}_{*}^{+}\right)$. The equilibrium fractions $\widetilde{\mathscr{Y}_{i}}$ are defined through the first derivatives of the entropies by (43)-(45) Therefore, $\mathscr{Y} \mapsto \widetilde{\mathscr{Y}}(\mathscr{Y})$ is continuous.

Since system (13) with source terms (42) is diagonal, each equation can be integrated between 0 and $t$ :

$$
\mathscr{Y}_{i}(t)=\left(\Phi_{i}(t)\right)^{-1} \mathscr{Y}_{i}(0)+\left(\Phi_{i}(t)\right)^{-1} \int_{0}^{t}\left(\frac{\Phi_{i}(s) \widetilde{\mathscr{Y}}(\mathscr{Y}(s))}{\lambda_{i}(s)}\right) d s,
$$

where we have for all $t$ :

$$
\Phi_{i}(t)=\exp \left(\int_{0}^{t} \frac{d \zeta}{\lambda(\zeta)}\right)
$$

Function $\Phi_{i}$ is such that for all $t$, we have: $\Phi_{i}(t)>0$ and $d_{t} \Phi_{i}(t)=\Phi_{i}(t) / \lambda_{i}(t)$. Provided that $\widetilde{\mathscr{Y}}_{i}(s) \geq 0$ for all $s \in[0, t]$, equation (46) clearly ensures that $\mathscr{Y}_{i}$ remains positive. Moreover, it should be noticed that:

$$
\int_{0}^{t}\left(\frac{\Phi_{i}(s)}{\lambda_{i}(s)}\right) d s=\int_{0}^{t} d_{s} \Phi_{i}(s) d s=\Phi_{i}(t)-1 .
$$

Hence, by using equation (46) and previous result (47), we obtain:

$$
1-\mathscr{Y}_{i}(t)=\left(\Phi_{i}(t)\right)^{-1}\left(1-\mathscr{Y}_{i}(0)\right)+\left(\Phi_{i}(t)\right)^{-1} \int_{0}^{t}\left(\frac{\Phi_{i}(s)\left(1-\widetilde{\mathscr{Y}_{i}}\right)}{\lambda_{i}(s)}\right) d s
$$

This equation clearly implies that if $1-\widetilde{\mathscr{Y}_{i}}(s) \geq 0$ for all $s \in[0, t]$, then $1-\mathscr{Y}_{i}(t) \geq 0$. We can conclude that if $\mathscr{Y}(0)$ lies in $[0,1]^{3}$ and if for all $s \in[0, t], \widetilde{\mathscr{Y}_{i}}(s)$ lies in $[0,1]^{3}$, then $\mathscr{Y}(t)$ lies in $[0,1]^{3}$.

Equilibrium states $\mathscr{Y}^{*}$ for system (13) with source terms (42) are such that:

$$
\mathscr{S}_{i}\left(\mathscr{Y}^{*}\right)=0 \Longleftrightarrow \mathscr{Y}_{i}^{*}=\widetilde{\mathscr{Y}}_{i}\left(\mathscr{Y}^{*}\right), i=1 . .3,
$$

which thanks to (43)-(45) reads:

$$
\begin{aligned}
\frac{P_{1}}{T_{1}}\left(\alpha_{1}^{*}, y_{1}^{*}, z_{1}^{*}\right) & =\frac{P_{2}}{T_{2}}\left(\alpha_{1}^{*}, y_{1}^{*}, z_{1}^{*}\right), \\
\frac{\mu_{1}}{T_{1}}\left(\alpha_{1}^{*}, y_{1}^{*}, z_{1}^{*}\right) & =\frac{\mu_{2}}{T_{2}}\left(\alpha_{1}^{*}, y_{1}^{*}, z_{1}^{*}\right), \\
\frac{1}{T_{1}}\left(\alpha_{1}^{*}, y_{1}^{*}, z_{1}^{*}\right) & =\frac{1}{T_{2}}\left(\alpha_{1}^{*}, y_{1}^{*}, z_{1}^{*}\right) .
\end{aligned}
$$

This exactly corresponds to the definition of $\overline{\mathscr{Y}}$ though (14)-(16), which is the unique solution of system (48)-(50). Source terms (42) is then associated with the same unique equilibrium state than source terms (21): $\mathscr{Y}^{*}=\overline{\mathscr{Y}}$.

We assume from now that each $\lambda_{i}$ is bounded for all $t$. This implies that the relaxation process for the fractions does not stop for a finite time, and that:

$$
\lim _{t \rightarrow \infty} \Phi_{i}(t)=+\infty
$$

Let us define $\mathscr{Y} \mapsto \mathscr{L}(\mathscr{Y})=s(\overline{\mathscr{Y}})-s(\mathscr{Y})$. In the previous sections, it has been shown that for source terms (42):

$$
\begin{array}{ll}
\text { (i) } & \mathscr{L}(\overline{\mathscr{Y}})=0 \text { and } d_{t} \mathscr{L}(\overline{\mathscr{Y}})=0, \\
\text { (ii }) & \forall \mathscr{Y} \neq \overline{\mathscr{Y}}, \mathscr{L}(\mathscr{Y})>0, \\
\text { (iii }) & \forall \mathscr{Y} \neq \overline{\mathscr{Y}}, d_{t} \mathscr{L}(\mathscr{Y})<0 .
\end{array}
$$

Therefore, $\mathscr{Y} \mapsto \mathscr{L}(\mathscr{Y})$ is a strict Lyapunov function for system (13) with source terms (42), which ensures that the equilibrium state defined by $\overline{\mathscr{Y}}$ is asymptotically stable. As $\mathscr{Y} \mapsto \widetilde{\mathscr{Y}}$ is continuous and $t \mapsto \mathscr{Y}(t)$ is continuous, this implies that $\widetilde{\mathscr{Y}}$ tends asymptotically to $\overline{\mathscr{Y}}$ when time increases. The vector $\widetilde{\mathscr{Y}}$ can thus be seen as a "local-in-time" equilibrium state that tends to the asymptotic equilibrium state $\overline{\mathscr{Y}}$.

\subsection{Relaxation process for the modified BGK source terms}

The aim of this section is to provide some remarks on the pressure and temperature relaxation process by following thermodynamical paths that ensure that nos mass transfer occurs. For the classical BGK source terms, such paths are obvious and correspond to a constant mass fractions: $y_{1}=\bar{y}_{1}$. For the modified source terms, these paths are such that: $y_{1}=\widetilde{y}_{1}\left(\alpha_{1}, z_{1}\right)$, where $\tilde{y}_{1}$ is implicitly defined by equation (44). Let us differentiate (44), we get:

$$
\sum_{k=1}^{k=2}\left(\frac{A_{k} \tau_{k}+B_{k} e_{k}}{y_{k}}\right) d y_{1}=\sum_{k=1}^{k=2}\left(\frac{A_{k} \tau_{k}}{\alpha_{k}}\right) d \alpha_{1}+\sum_{k=1}^{k=2}\left(\frac{B_{k} e_{k}}{z_{k}}\right) d z_{1},
$$


with:

$$
A_{k}=e_{k} \partial_{e_{k} \tau_{k}}^{2}\left(s_{k}\right)+\tau_{k} \partial_{\tau_{k} \tau_{k}}^{2}\left(s_{k}\right), \quad \text { and } \quad B_{k}=e_{k} \partial_{e_{k} e_{k}}^{2}\left(s_{k}\right)+\tau_{k} \partial_{e_{k} \tau_{k}}^{2}\left(s_{k}\right) .
$$

When considering the Hessian matrix of $s_{k}$, we have:

$$
\left(\begin{array}{c}
A_{k} \\
B_{k}
\end{array}\right)=\nabla_{\left(\tau_{k}, e_{k}\right)}^{2} s_{k}\left(\begin{array}{c}
\tau_{k} \\
e_{k}
\end{array}\right) .
$$

The phasic entropies are strictly concave, so that the sum on the left hand side of equation (51) is strictly negative because:

$$
A_{k} \tau_{k}+B_{k} e_{k}=\left(\begin{array}{ll}
\tau_{k} & e_{k}
\end{array}\right) \cdot\left(\begin{array}{c}
A_{k} \\
B_{k}
\end{array}\right)=\left(\begin{array}{ll}
\tau_{k} & e_{k}
\end{array}\right) \cdot \nabla_{\left(\tau_{k}, e_{k}\right)}^{2} s_{k}\left(\begin{array}{c}
\tau_{k} \\
e_{k}
\end{array}\right)<0 .
$$

So that $d y_{k}$ is correctly defined through equation (51). From now it is assumed that $y_{1}=\widetilde{y}_{1}\left(\alpha_{1}, z_{1}\right)$. By considering a function $\phi_{k}:\left(\tau_{k}, e_{k}\right) \mapsto \phi_{k}\left(\tau_{k}, e_{k}\right)$, the counterpart of equation (24) is here:

$$
\begin{aligned}
d_{t} \phi_{k} & =\left(\partial_{\tau_{k}}\left(\phi_{f}\right) \frac{\tau_{k}}{\alpha_{k}}-\frac{\mathscr{A}_{\alpha}}{\mathscr{A}_{y}}\left(\partial_{\tau_{k}}\left(\phi_{f}\right) \frac{\tau_{k}}{y_{k}}+\partial_{e_{k}}\left(\phi_{f}\right) \frac{e_{k}}{y_{k}}\right)\right) d_{t} \alpha_{k} \\
& +\left(\partial_{e_{k}}\left(\phi_{f}\right) \frac{e_{k}}{z_{k}}-\frac{\mathscr{A}_{z}}{\mathscr{A}_{y}}\left(\partial_{\tau_{k}}\left(\phi_{f}\right) \frac{\tau_{k}}{y_{k}}+\partial_{e_{k}}\left(\phi_{f}\right) \frac{e_{k}}{y_{k}}\right)\right) d_{t} z_{k},
\end{aligned}
$$

where

$$
\mathscr{A}_{\alpha}=\sum_{k=1}^{k=2}\left(\frac{A_{k} \tau_{k}}{\alpha_{k}}\right), \quad \mathscr{A}_{y}=\sum_{k=1}^{k=2}\left(\frac{A_{k} \tau_{k}+B_{k} e_{k}}{y_{k}}\right), \quad \mathscr{A}_{z}=\sum_{k=1}^{k=2}\left(\frac{B_{k} e_{k}}{z_{k}}\right) .
$$

Thanks to (52), we obviously have $\mathscr{A}_{y}<0$. Then, by setting successively $\phi_{k}=1 / T_{k}=\partial_{e_{k}}\left(s_{k}\right)_{\mid \tau_{k}}$ and $\phi_{k}=P_{k} / T_{k}=$ $\partial_{\tau_{k}}\left(s_{k}\right)_{\mid e_{k}}$ in equation (53) one obtains:

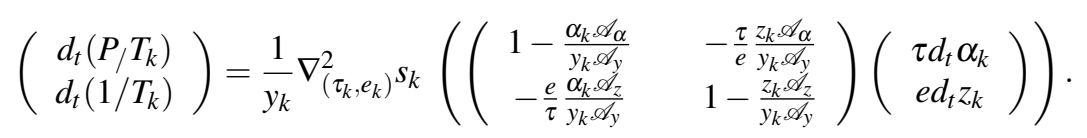

It should be noted that equation (25) is recovered by setting $\mathscr{A}_{\alpha}=\mathscr{A}_{z}=0$ (with $\mathscr{A}_{y}<0$ ) in equation (54), the latter corresponds to $d_{t} y_{1}=0$. But in general, the second matrix on the right hand side of equation (54) is not equal to the identity matrix and the paths followed by the difference of the phasic pressure and the difference of the phasic temperature are different than for the classical BGK source terms. Let us denote $\mathscr{D}_{k}$ this matrix:

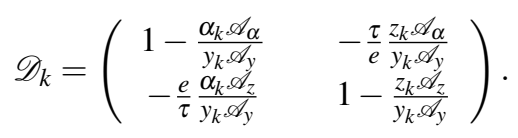

The matrix $\mathscr{D}_{k}$ possesses two real eigenvalues:

$$
r_{1, k}=1 \quad \text { and } \quad r_{2, k}=1-\frac{\alpha_{k} \mathscr{A}_{\alpha}+z_{k} \mathscr{A}_{z}}{y_{k} \mathscr{A}_{y}} .
$$

The second eigenvalue, $r_{2, k}$, depends on the thermodynamical state defined by $\alpha$ and $z$. Since we have the relation:

$$
\alpha_{k} \mathscr{A}_{\alpha}+z_{k} \mathscr{A}_{z}=A_{k} \tau_{k}+B_{k} e_{k},
$$

the eigenvalue $r_{2, k}$ can be rewritten:

$$
r_{2, k}=1-\frac{\left(A_{k} \tau_{k}+B_{k} e_{k}\right) / y_{k}}{\mathscr{A}_{y}} .
$$

and thanks to (52) we can deduce that $\left.r_{2, k} \in\right] 0,1$ [. Moreover, we have $r_{2,1}+r_{2,2}=1$. The eigenvectors $V_{1, k}$ and $V_{2, k}$ that are respectively associated with $r_{1, k}$ and $r_{2, k}$ are:

$$
V_{1, k}={ }^{t}\left(\tau / \alpha_{k},-e / z_{k}\right) \quad \text { and } \quad V_{2, k}={ }^{t}\left(\tau \mathscr{A}_{\alpha}, e \mathscr{A}_{z}\right) .
$$

It should be quoted that the eigenvector $V_{2, k}$ is the same for both phases. The two eigenvectors are linearly independent since:

$$
\operatorname{det}\left(V_{1, k}, V_{2, k}\right)=\frac{\tau e}{\alpha_{k} z_{k}}\left(A_{k} \tau_{k}+B_{k} e_{k}\right)<0
$$

The matrix $\mathscr{D}_{k}$ is then diagonalizable on $\mathbb{R}^{2}$, but it is not symmetric. The time evolution of the pressure and temperature differences is then obtained by combining equations (54):

$$
\left(\begin{array}{c}
d_{t}\left(P_{1} / T_{1}-P_{2} / T_{2}\right) \\
d_{t}\left(1 / T_{1}-1 / T_{2}\right)
\end{array}\right)=\left(\nabla_{\left(\tau_{1}, e_{1}\right)}^{2} s_{1} \frac{\mathscr{D}_{1}}{y_{1}}+\nabla_{\left(\tau_{2}, e_{2}\right)}^{2} s_{2} \frac{\mathscr{D}_{2}}{y_{2}}\right)\left(\begin{array}{c}
\tau d_{t} \alpha_{1} \\
e d_{t} z_{1}
\end{array}\right)
$$


Unfortunately, since $\mathscr{D}_{k}$ is not symmetric, the counterpart of inequality (28) can not be obtained from (55). This makes the analysis of the relaxation process even more complex than for the classical BGK source terms.

Remark 5. When both phases are described by a Perfect Gas EoS, some specific results can be obtained. In particular, the equilibrium fractions are very close to those obtained in section 4.4, see equations (32) and (33), except that they depend on $\mathscr{Y}$. They are obtained from equations (43)-(45):

$$
\begin{array}{r}
\widetilde{\alpha}_{1}=\frac{y_{1} \delta_{1}}{y_{1} \delta_{1}+y_{2} \delta_{2}}, \\
\widetilde{z}_{1}=\frac{y_{1} C_{v, 1}}{y_{1} C_{v, 1}+y_{2} C_{v, 2}}, \\
\left.\gamma_{1} C_{V, 1}-s_{1}\left(\frac{\alpha_{1} \tau}{\widetilde{y}_{1}}, \frac{z_{1} e}{\widetilde{y}_{1}}\right)=\gamma_{2} C_{V, 2}-s_{2}\left(\frac{\alpha_{2} \tau}{\left(1-\widetilde{y}_{1}\right)}, \frac{z_{2} e}{\left(1-\widetilde{y}_{1}\right.}\right)\right) .
\end{array}
$$

For these Perfect Gas EoS, it should be noted that $\widetilde{\alpha}_{1}$ and $\widetilde{z}_{1}$ only depend on $y_{1}$ whereas $\widetilde{y}_{1}$ depends on $\alpha_{1}$ and $z_{1}$. This is due to the non-linearity of the chemical potential. On the contrary to the source terms of section 4.4, the thermodynamical path, $y_{1}=\widetilde{y}_{1}\left(\alpha_{1}, z_{1}\right)$, for which no mass transfer occurs can not be explicitly written. The analysis of the sole pressure and temperature relaxations is then more tricky even for Perfect Gas EoS.

\section{Conclusion}

A modified form of BGK source terms has been proposed for modeling two-phase flows with thermodynamical disequilibrium. For these source terms, three independent time-scales manage the return to the thermodynamical equilibrium while remaining in agreement with the second law of thermodynamics. The relaxation process can then be defined with additional degrees of freedom. Nonetheless, relaxation process remains complex and further studies should be carried in order to analysis in details the pressure and temperature relaxation. In particular, with the BGK source terms of sections 4 and 5, it is not an easy task to impose instantaneous relaxation of the pressure or the temperature for general EoS.

In a numerical point of view, both classical and modified BGK source terms have advantages and drawbacks depending on the numerical strategy. For the classical BGK source terms, the simulation of the ODE system relies on the unique computation of the asymptotic equilibrium state through a $3 \times 3$ non-linear system. Then, if the time scale can be explicitly integrated, the overall integration of the classical source terms is exact [20, 21]. On the contrary, for the modified BGK source terms, the ODE system involves a "local-in-time" thermodynamical equilibrium which tends towards the asymptotic equilibrium. The computation of this "local-in-time" equilibrium state is based on three independent non-linear equations. Hence, when considering a fractional step approach for which the ODE system for relaxation process is solved using a unique time-step, modified BGK form can be preferred provided that the time-step is much smaller than the characteristic time for return to equilibrium. On the contrary, if several time steps are involved for solving the ODE system, the classical BGK source terms are less CPU-consuming since the thermodynamical equilibrium is only computed once at the beginning of the integration.

\section{References}

[1] M. Baer and J. Nunziato. A two-phase mixture theory for the Deflagration-To-Detonation transition (DDT) in reactive granular materials. Journal of Multiphase Flows, 12:861-889, 1986.

[2] T. Barberon and P. Helluy. Finite volume simulation of cavitating flows. Computers and Fluids, 34(7):832$858,2005$.

[3] H. Boukili and J.-M. Hérard. Relaxation and simulation of a barotropic three-phase flow model. ESAIM: Mathematical Modelling and Numerical Analysis, 53(3):1031-1059, 2019.

[4] H. Boukili and J.-M. Hérard. Simulation and preliminary validation of a three-phase flow model with energy. Computers \& Fluids, 221:104868, 2021.

[5] A. Chiapolino, P. Boivin, and R. Saurel. A simple and fast phase transition relaxation solver for compressible multicomponent two-phase flows. Computers \& Fluids, 150:31-45, 2017.

[6] F. Coquel, T. Gallouët, J.-M. Hérard, and N. Seguin. Closure laws for a two-fluid two-pressure model. Comptes Rendus Mathematique, 334(10):927-932, 2002.

[7] P. Downar-Zapolski, Z. Bilicki, L. Bolle, and J. Franco. The non-equilibrium relaxation model for onedimensional flashing liquid flow. International Journal of Multiphase Flow, 22(3):473-483, 1996. 
[8] G. Faccanoni, S. Kokh, and G. Allaire. Modelling and simulation of liquid-vapor phase transition in compressible flows based on thermodynamical equilibrium. ESAIM: Mathematical Modelling and Numerical Analysis, 46(5):1029-1054, 2012.

[9] G. Faccanoni and H. Mathis. Admissible Equations of State for Immiscible and Miscible Mixtures. ESAIM: Proceedings and Surveys, 66:1-21, 2019.

[10] E. Faucher, J.-M. Hérard, M. Barret, and C. Toulemonde. Computation of flashing flows in variable crosssecglimm1998two,tion ducts. International Journal of Computational Fluid Dynamics, 13(3):365-391, 2000.

[11] S. Gavrilyuk and R. Saurel. Mathematical and numerical modeling of two-phase compressible flows with micro-inertia. J. Comput. Phys., 175(1):326-360, Jan. 2002.

[12] H. Ghazi. Modélisation d'écoulements compressibles avec transition de phase et prise en compte des états métastables. Phd thesis, Université de Nantes, 2018.

[13] H. Ghazi, F. James, and H. Mathis. Vapour-liquid phase transition and metastability. ESAIM: Proceedings and Surveys, 66:22-41, 2019.

[14] H. Ghazi, F. James, and H. Mathis. A nonisothermal thermodynamical model of liquid-vapor interaction with metastability. Discrete and Continuous Dynamical Systems - B, 26(5):2371-2409, 2021.

[15] J. Glimm, D. Saltz, and D. Sharp. Two-pressure two-phase flow. In Advances In Nonlinear Partial Differential Equations And Related Areas: A Volume in Honor of Professor Xiaqi Ding, pages 124-148. World Scientific, 1998.

[16] P. Helluy, O. Hurisse, and L. Quibel. Assessment of numerical schemes for complex two-phase flows with real equations of state. Computers \& Fluids, 196:104347, 2020.

[17] J.-M. Hérard and O. Hurisse. Computing two-fluid models of compressible water-vapour flows with mass transfer. In 42nd AIAA Fluid Dynamics Conference and Exhibit, page 2959, 2012.

[18] J.-M. Hérard and O. Hurisse. A fractional step method to compute a class of compressible gas-liquid flows. Computers \& Fluids, 55:57-69, 2012.

[19] J.-M. Hérard, O. Hurisse, and L. Quibel. A four-field three-phase flow model with both miscible and immiscible components. ESAIM: Mathematical Modelling and Numerical Analysis, 2020.

[20] O. Hurisse. Application of an homogeneous model to simulate the heating of two-phase flows. International Journal on Finite Volumes, 11:http://www.latp.univ-mrs.fr/IJFV/spip.php?article52, May 2014.

[21] O. Hurisse. Numerical simulations of steady and unsteady two-phase flows using a homogeneous model. Computers and Fluids, 152:88-103, July 2017.

[22] O. Hurisse. Various choices of source terms for a class of two-fluid two-velocity models. ESAIM: Mathematical Modelling and Numerical Analysis, 55(2):357-380, 2020.

[23] O. Hurisse and L. Quibel. A homogeneous model for compressible three-phase flows involving heat and mass transfer. ESAIM: Proceedings and Surveys, 66:81-108, 2019.

[24] O. Hurisse and L. Quibel. Simulations of liquid-vapor water flows with non-condensable gases on the basis of a two-fluid model. Applied Mathematical Modelling, 2021.

[25] H. Jin, J. Glimm, and D. Sharp. Compressible two-pressure two-phase flow models. Physics letters A, 353(6):469-474, 2006.

[26] J. Jung. Numerical simulations of two-fluid flow on multicores accelerator. Phd thesis, Université de Strasbourg, Oct. 2013.

[27] A. K. Kapila, R. Menikoff, J. B. Bdzil, S. F. Son, and D. S. Stewart. Two-phase modeling of deflagrationto-detonation transition in granular materials: Reduced equations. Physics of Fluids, 13(10):3002-3024, 2001 .

[28] Y. Liu. Contribution to the verification and the validation of an unsteady two-phase flow model. Phd thesis, Aix-Marseille Université, Sept. 2013.

[29] H. Lochon. Modélisation et simulation d'écoulements transitoires eau-vapeur en approche bifluide. Phd thesis, Aix Marseille Université, 2016.

[30] H. Mathis. A thermodynamically consistent model of a liquid-vapor fluid with a gas. ESAIM: Mathematical Modelling and Numerical Analysis, 53(1):63-84, 2019. 
[31] L. Quibel. Simulation of water-vapor two-phase flows with non-condensable gas. Phd thesis, Université de Strasbourg, Sept. 2020.

[32] B. Stewart and B. Wendroff. Two-Phase Flows : models and methods. Journal of Computational Physics, 56(7):363-409, 1984. 